\title{
Las percepciones sobre la educación en un sistema excluyente: un amor no del todo correspondido
}

\section{Perceptions of education in a discriminatory system:} an unreciprocated love

\author{
Judith Zubieta García ${ }^{1}$ \\ Universidad Nacional Autónoma de México (UNAM) \\ zubieta@unam.mx
}

\begin{abstract}
Resumen: El sector educativo mexicano ha recibido grandes aportaciones del erario público y ha merecido destacadas menciones en los planes nacionales de desarrollo elaborados por las administraciones federales en turno. Sus resultados, sin embargo, siguen siendo cuestionados no sólo porque no se ha podido garantizar el derecho de todos los mexicanos a la educación, sino también porque la calidad de los servicios que ofrece ha sido deficiente. Se parte del análisis de las grandes inequidades educativas que prevalecen en el país y éstas se contrastan con el alto valor que la población sigue atribuyendo a la educación. Además de presentar la complejidad de la evaluación de la calidad educativa y de reconocer que son múltiples los factores asociados a la exclusión, llama la atención que la percepción general de la población sobre la educación sea tan positiva, aunque se reconozcan algunas fallas en el funcionamiento del Sistema Educativo Nacional. Ante la proximidad de un cambio de gobierno federal, el texto propone algunas consideraciones para el diseño de nuevas políticas educativas.
\end{abstract}

Palabras Clave: Inequidad educativa, percepciones sobre educación, políticas educativas.

Abstract: The Mexican educational sector has received large contributions from the public purse and has earned prominent mentions in national development plans drawn up by federal authorities. Its results are nonetheless being contested not only because it has not been able to guarantee the right of all Mexicans to education, but also because the quality of the services offered is questionable. This

${ }^{1}$ La autora agradece el apoyo del programa PASPA-DGAPA de la UNAM sin el cual no habría sido posible realizar la estancia sabática durante la cual se preparó este artículo. 
paper contrasts major educational inequities prevailing in the country with the high value that the population still attributes to education. While recognizing that there are multiple factors associated to educational exclusion and the quality of education, it is striking that the general perception of the population regarding education is positive, even though some faults are acknowledged in the operation of the National Education System. Due to the proximity of a change in the Government, the paper elaborates some considerations for the design of new educational policies.

Keywords: Educational inequity, Perceptions on education, educational policies. 


\section{INTRODUCCIÓN}

De tiempo atrás, el Sistema Educativo Nacional (SEN) se enfrenta a múltiples paradojas. Una de ellas involucra la buena opinión que los mexicanos tienen de un sistema que se ha caracterizado por ser excluyente. Este sistema —organizado y estructurado para dar cabida a todos los mexicanos y así cumplir el mandato derivado del Artículo $3^{\circ}$ de la Constitución Política de los Estados Unidos Mexicanos- ha sido a la vez discriminatorio. ${ }^{2}$

Efectivamente, el SEN se ha caracterizado más por reproducir las desigualdades sociales que por contribuir a la movilidad social. La exclusión afecta principalmente a individuos en situación de pobreza, indígenas, discapacitados y, en general, a poblaciones vulnerables. Las condiciones de acceso también han sido inequitativas entre regiones y al interior de ellas. Entre los grupos sociales que han sufrido esta marginación, vale la pena señalar a las mujeres, quienes hasta la segunda mitad del siglo XX constituyeron más de la mitad de esa población vulnerable, aunque paulatinamente han ido obteniendo mayores niveles de equidad, al menos en términos de su acceso al sistema.

Grandes son las desigualdades que han predominado entre la población mexicana y por ello grandes son los retos que enfrenta el SEN, no sólo en materia de su cobertura. ${ }^{3}$ En un país en el que cinco de cada diez habitantes es menor de 29 años (es decir, casi la mitad de la población está en edad de escolarizarse), la complejidad aumenta. ${ }^{4} \mathrm{El}$ panorama se complica más si se considera que entre la población que se encuentra en situación de pobreza extrema, sólo $20 \%$ de los jóvenes de 12 a 14 años y poco más de la mitad de los de 15 a 17 asisten a la escuela. ${ }^{5}$

Los obstáculos no sólo se expresan en términos de marginación y de rezago, sino que también existe un problema muy serio de calidad porque la población marginada asiste a escuelas con severas carencias en materia de infraestructura, con un profesorado generalmente mal pagado y formado con limitaciones, por lo que la calidad educativa dista mucho de la que se debería ofrecer a todos, especialmente a los segmentos más necesitados.

2 Este artículo señala la obligación del Estado de «garantizar la calidad en la educación obligatoria de manera que los materiales y los métodos educativos, la organización escolar, la infraestructura educativa y la idoneidad de los docentes y los directivos garanticen el máximo logro de aprendizaje de los educandos.»

${ }^{3}$ Para un análisis completo de la cobertura del SEN, véase: Rodríguez, R. e I. Ordorika (2012).

${ }^{4}$ En este texto no se considera la educación para la vida y el trabajo —o aprendizaje a lo largo de la vida - en la que las edades tradicionalmente asociadas a la escolaridad se vuelven irrelevantes.

${ }^{5}$ Fuente: INEE (2016a). 
En un país en el que las políticas educativas han tenido objetivos planteados formalmente en términos de reducir el rezago y aumentar la calidad educativa, pareciera importante conocer la percepción de los mexicanos sobre la educación e indagar si, pese a que las mujeres se encontraron en situación de desventaja en relación a esas oportunidades o precisamente por ello, existen diferencias de género.

A grandes rasgos, se reconoce que los factores que la población identifica como determinantes de la exclusión educativa, así como la valoración que hombres y mujeres dan a la educación, pudieran estar influenciados por sus percepciones sobre el mercado de trabajo y por la relación que pueden identificar entre éste y lo aprendido en la escuela.

De esta manera, este texto se divide en tres partes. En la primera se analizan brevemente algunas cifras del SEN, para posteriormente ubicarlas dentro del marco normativo y la política educativa que lo ha regido en los últimos años. Esto permite abordar el tema de la calidad de la educación, otra de las deudas que el gobierno mexicano tiene con la sociedad. En la segunda parte se presentan los resultados de una encuesta mediante la cual logramos capturar, entre otras variables, las percepciones que tienen los mexicanos sobre la educación. Además de la discusión de dichos hallazgos y de construir algunas hipótesis para explicar las paradojas identificadas, en la tercera parte se delinean líneas de política educativa que podrán contribuir a enfrentar con mayor éxito los grandes retos del SEN.

\section{UNA COBERTURA CON FALTANTES}

Además de brindar educación de calidad a un número creciente de mexicanos, un sistema educativo debe dar respuesta a las necesidades provenientes de la diversidad étnica, cultural y lingüística que caracteriza a un país como México, además de incorporar a los grupos que se encuentran dispersos geográficamente ${ }^{6}$.

En términos generales, la tasa de cobertura que estima la Secretaría de Educación Pública (SEP; 2016) para cada nivel educativo en el ciclo 2016-2017 es la siguiente: educación preescolar (3 a 5 años de edad), 62.3; educación primaria (6 a 11 años), 104.2; educación secundaria (12 a 14 años), 98.7; educación media superior (15 a 17), 70; y educación superior (18 a 22), 27.2, excluyendo posgrado ${ }^{7}$.

${ }^{6}$ El 23\% de la población mexicana habita en localidades de menos de 2,500 habitantes y un 72.4\% del total de localidades en el país tiene una población de menos de 100 habitantes (INEGI, 2015).

7 Fuente: SEP (2016). La tasa superior a 100 indica que no sólo atiende a población en el grupo etario correspondiente, sino que también incorpora alumnos de cohortes anteriores que han reprobado o que abandonaron temporalmente los estudios. 
La escolaridad promedio, como puede observarse en la Gráfica 1, ha ido en aumento. Los últimos datos del Instituto Nacional para la Evaluación de la Educación (INEE) correspondientes a 2015 estiman que la escolaridad promedio en el país asciende a 9.2 años, promedio que aun cuando las diferencias por sexo son casi imperceptibles, ocultan las prevalecientes entre regiones, estados, el ámbito urbano y rural, o entre estratos socioeconómicos.

\section{GRÁFICA 1}

México: Escolaridad promedio de la población de 15 años y más, 1970-2015

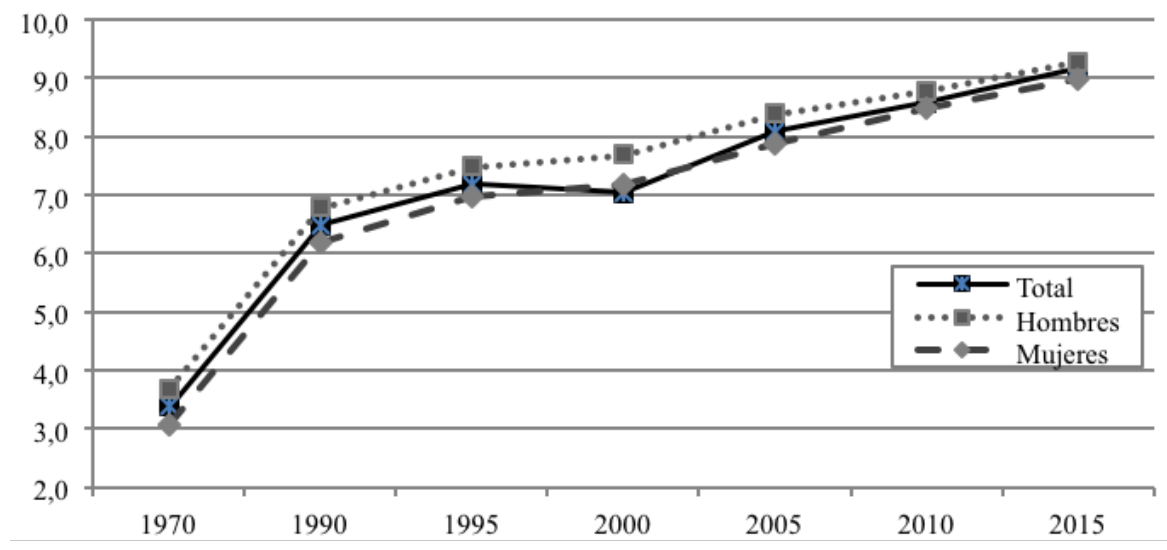

Fuente: INEE (2015), Panorama Educativo de México.

A pesar de los incrementos en la escolaridad promedio, el analfabetismo persiste y afecta a más de 5 millones de mexicanos. Según datos oficiales, el país tiene cerca de 6 millones de mexicanos que que hablan diversas lenguas indígenas y de ellos, pocos hablan español ${ }^{8}$. Más allá del énfasis que se haya puesto en la formación del magisterio a la atención de las demandas educativas específicas de estos grupos e independientemente de si se cuenta con un número suficiente de maestras y maestros en las regiones o estados con mayor concentración de población originaria que sean hablantes de esas lenguas, surge la duda sobre la pertinencia de tener planes de estudio nacionales y sistemas educativos homogéneos cuando las condiciones de vida y la cultura son tan heterogéneas.

${ }^{8}$ De acuerdo con cifras del INEE (2017), en 2015 la población indígena superaba los 12 millones; de ellos, poco más de 7.1 millones habla alguna lengua indígena y uno de cada diez es monolingüe. 
CUADRO 1

Escolaridad media de la población mexicana, 2015

\begin{tabular}{lccccc}
\hline \multirow{2}{*}{$\begin{array}{c}\text { Subpoblación } \\
\text { seleccionada }\end{array}$} & \multicolumn{5}{c}{ Escolaridad media según grupo de edad } \\
\cline { 2 - 6 } & $\mathbf{1 5}$ a $\mathbf{~ 4 4}$ & $\mathbf{2 5}$ a $\mathbf{3 4}$ & $\mathbf{5 5}$ a $\mathbf{6 4}$ & $\mathbf{2 5}$ o más & $\mathbf{1 5}$ o más \\
\hline Población total & 10,1 & 10,6 & 7,4 & 8,9 & 9,2 \\
Hombres & 10,0 & 10,6 & 8,0 & 9,1 & 9,3 \\
Mujeres & 10,3 & 10,7 & 6,9 & 8,6 & 9,0 \\
Tamaño de localidad & & & & & \\
Rural & 9,0 & 8,3 & 3,9 & 5,9 & 6,7 \\
Semiurbana & 9,8 & 9,8 & 5,8 & 7,7 & 8,3 \\
Urbana & 10,7 & 11,6 & 8,8 & 10,1 & 10,2 \\
\hline
\end{tabular}

Fuente: INEE (2015), Panorama Educativo de México.

A pesar de las variaciones que se observan en el Cuadro 1, se puede decir que la escolaridad promedio de la población mexicana está estimada actualmente en poco más de 9 años, lo cual indica que se tiene un rezago educativo de importancia, generalmente acompañado de altas tasas de abandono y de reprobación. Algunos autores consideran que más del 40\% de los mexicanos de 15 años y más está en esta condición ${ }^{9}$.

Si bien la escolaridad promedio se ha incrementado, la posibilidad de permanecer en el sistema es baja y disminuye conforme se avanza a niveles educativos superiores, especialmente en el caso de poblaciones vulnerables. Aunque resulte satisfactorio que la cobertura en educacion básica sea ya prácticamente universal, persisten carencias en el caso de la educación preescolar, particularmente entre la población que habita en zonas rurales, grupos indígenas y personas que se encuentran en condiciones de pobreza. Se suma a esta característica que en la educación básica, al igual que en la media superior, las cifras revelan un serio problema de deserción y abandono escolar.

${ }^{9}$ Véase: Fraustro, A. (2017) 


\section{CUADRO 2}

Tasas de Rezago en la escolarización por grupos de edad, 2015

\begin{tabular}{lccc}
\hline \multicolumn{1}{c}{ Población } & 15 o más & $\mathbf{1 5 - 2 4}$ & $\mathbf{5 5}$ a 64 \\
\hline Total & 35,4 & 19,9 & 58,4 \\
Hombres & 34,4 & 21,5 & 54,2 \\
Mujeres & 36,3 & 18,4 & 62,2 \\
Tamaño de localidad & & & \\
Rural & 56,4 & 30,7 & 86,5 \\
Semiurbana & 42,1 & 22,8 & 72,2 \\
Urbana & 26,8 & 15,1 & 46,8 \\
\hline
\end{tabular}

Nota: Número de personas con 15 años o más que no tienen educación secundaria completa por cada cien de la población total en el mismo grupo de edad.

Fuente: INEE (2015), Panorama Educativo de México.

Al igual que en el caso del analfabetismo, el problema del rezago es multifactorial; sin embargo, es claro que grandes desigualdades sociales contribuyen a reproducir mayores condiciones de inequidad en el acceso a la educación.

En términos de escolaridad, la exclusión femenina se refleja en un mayor número de mujeres en los niveles iniciales del SEN. No obstante, este patrón general, las mujeres no sólo han logrado el acceso a la educación básica, sino que, además, reportan tasas de egreso superiores a las de los varones ${ }^{10}$. Esta situación deja de ser verdadera en niveles más elevados, empezando por la Educación Media Superior (también llamada Bachillerato), donde las tasas de absorción de las mujeres son menores y las de abandono, mayores ${ }^{11}$.

10 Véase: INEE (2016b), Panorama Educativo de México, p. 291.

${ }^{11}$ El análisis de la educación media superior se ha complicado a partir de que se decretó su obligatoriedad en 2012. A partir de esa fecha, se incorporaron grandes grupos de personas que habían concluido la secundaria en generaciones previas. No obstante, el INEE sigue reportando tasas de absorción femenina inferiores a las de los varones en este nivel educativo. 


\section{GRÁFICA 2}

Escolaridad de la población de 15 años y más

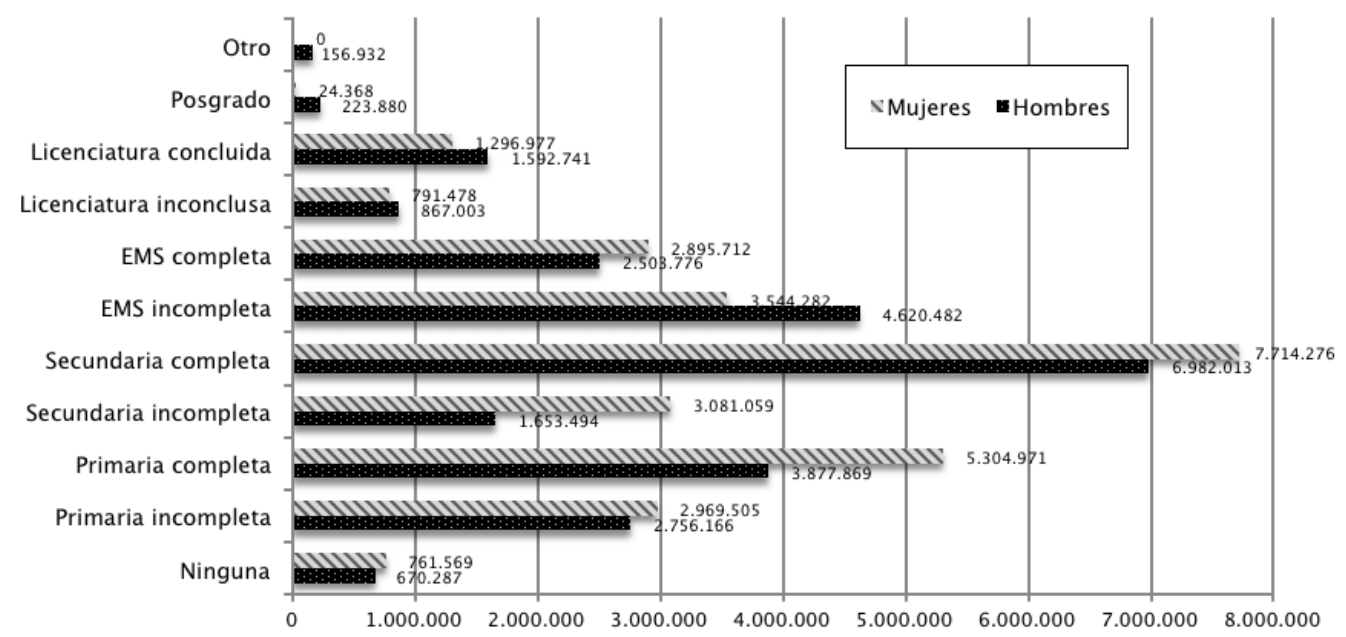

Fuente: CEPAL (2013).

No debe soslayarse en este análisis que diversas iniciativas instrumentadas por el gobierno federal en los últimos veinte años han favorecido incrementos en la absorción y en la cobertura, pero tampoco debe menospreciarse que al tiempo que no se ha abatido el rezago educativo, particularmente en los niveles medio superior y superior, tampoco se ha logrado erradicar el analfabetismo, por no mencionar el tema de la calidad, que sigue siendo baja en todos los niveles educativos.

Las evaluaciones nacionales sobre la calidad de los servicios educativos de primaria y secundaria han demostrado la existencia de una relación estrecha entre los resultados que obtienen los alumnos en pruebas estandarizadas y las condiciones del entorno socioeconómico de la escuela, lo que implica la prevalencia de una enorme heterogeneidad, segmentación y desigualdad ${ }^{12}$.

Dado que la educación es un derecho que se encuentra plasmado en la Carta Magna, resulta paradójico que el propio Estado no haya asumido explícitamente el compromiso de disminuir el déficit educativo y que tampoco haya diseñado instrumentos y mecanismos para lograrlo. Ésta es una evidencia más de la gran distancia que existe entre los

12 Véase: Rodríguez y Ordorika (2012: 205). 
marcos normativos, las políticas gubernamentales y la realidad en la que subsisten millones de mexicanos.

Cada vez se vuelve más urgente la necesidad de combatir el rezago educativo; en particular, el de quienes no logran terminar la educación básica y el de quienes no logran transitar al nivel inmediato superior. Según lo mandata la Constitución Política de los Estados Unidos Mexicanos, es impostergable ampliar el acceso a la Educación Media Superior y disminuir las grandes desigualdades que se registran entre las entidades federativas y al interior de ellas.

Estos son, en resumen, algunos de los grandes retos que enfrenta el SEN, los mismos que deberán enfrentarse de manera apremiante en la próxima administración federal, que tomará posesión a finales de 2018.

\section{CALIDAD Y LOGRO ESCOLAR}

Hablar de la calidad educativa se ha traducido en sinónimo de hablar de mecanismos de evaluación y de afectación al salario. Distintos esquemas de evaluación se han puesto en marcha atendiendo distintas definiciones del concepto de calidad sin que, hasta ahora, se haya contemplado la posibilidad de evaluar la evaluación. Se ha intentado evaluar a los actores (docentes, directivos y alumnado), a las instituciones, los contenidos y los materiales didácticos, pero estas evaluaciones no han considerado la naturaleza sistémica de los procesos de enseñanza y de aprendizaje y, en lugar de servir como referentes para la mejora, han tenido como resultado punitivo la descalificación.

En el caso de las y los alumnos, los resultados de distintas evaluaciones estandarizadas no han reportado mejorías. Un ejemplo es el pobre desempeño en el Programa para la Evaluación Internacional de Estudiantes (PISA), donde los resultados se encuentran sistemáticamente por debajo de la media de la OCDE y México nunca ha logrado resultados favorables ni siquiera al compararse consigo mismo. Aún en el caso del Plan Nacional para la Evaluación de los Aprendizajes (PLANEA), puesto en marcha en el ciclo escolar 2014-2015 para evaluar el logro educativo en la educación básica y media superior, los resultados tampoco han sido alentadores, lo cual evidencia que este tipo de pruebas estandarizadas son cuestionables para una sociedad tan heterogénea y desigual como la mexicana, además de sus efectos perniciosos en el propio sistema educativo.

No nada más se debe intentar el aumento en el volumen de la población que asiste a la escuela, también debiera existir un compromiso por incrementar la calidad de la educación que se ofrece. Las enormes desigualdades que lastiman a los más necesitados cada vez reflejan más el sello que ha caracterizado a un sistema que durante décadas ha 
recibido vastos recursos del erario público y que, evidentemente, no ha podido, sabido o querido canalizar para asegurar su mejora, independientemente de los indicadores que se utilicen para medirla.

\section{LA POCA EFECTIVIDAD DE LAS POLÍTICAS EDUCATIVAS}

El discurso oficial ha tenido como constante, desde la creación de la hoy Secretaría de Educación Pública, la preocupación por la cobertura; a mediados del siglo pasado, a ésta se agregó un nuevo componente: el de la calidad, que en la práctica se ha interpretado pobremente como resultante de un proceso de evaluación, con las limitaciones que brevemente ya se han comentado.

Un rápido análisis de los planes nacionales de desarrollo (PND) a partir de 1995, permite advertir la existencia de un elemento presente en todos: la insistencia en la calidad educativa —y, como era de esperarse, el de la evaluación de la calidad- aunque con distintos énfasis y matices ${ }^{13}$. En el PND correspondiente al periodo 1995-2000, se hace el planteamiento de avanzar hacia un desarrollo social, ampliando la dotación de servicios de calidad. En el siguiente (2001-2006), el objetivo fue formulado en términos de desarrollo social y humano, elevando la calidad y la cobertura educativas. El plan subsecuente (2007-2012) propuso la igualdad de oportunidades y, en materia educativa, añadió a la cobertura el componente de calidad, asociándola con una preocupación por su relevancia para el sector productivo. El PND correspondiente a la administración del Presidente Enrique Peña Nieto (2013-2018) estableció como objetivo «México con educación de calidad», señalando al 'capital humano' como factor esencial para llevar al país «a su máximo potencial» y tratando de poner en práctica una reforma en la educación obligatoria ${ }^{14}$.

No huelga dejar asentado que la mal llamada Reforma Educativa —emprendida por el gobierno actual - en realidad ha sido una reforma de carácter político, en la que el énfasis

${ }^{13}$ De acuerdo a lo que señala la Ley del INEE, se entiende por Calidad de la educción a la cualidad de un sistema educativo que integra las dimensiones de relevancia, pertinencia, equidad, eficiencia, eficacia, impacto y suficiencia.

${ }^{14}$ Dado que éste es el primer Plan en el que se habla de capital humano, conviene recordar a Holland $(2009 ; 335)$ cuando señala que una de las críticas más importantes a la teoría del capital social, además de las múltiples dificultades que el propio concepto presenta, es que ha permitido que se ignoren las diferencias de género, raza, etnia, y otros aspectos de la diversidad. Precisamente eso es lo que han hecho estas políticas públicas en materia educativa y los resultados de haberlas puesto en práctica son los que se presentan en la primera parte del presente texto. 
estuvo centrado en la modificación de las relaciones entre el gobierno federal y el Sindicato de Trabajadores de la Educación; es decir, los maestros que, agremiados en este Sindicato, dejaron de serle útiles al partido gobernante, a pesar de haberle brindado jugosos dividendos. $\mathrm{Al}$ ignorar otros factores de gran trascendencia (como la formación que reciben y las condiciones en las que trabajan, lo mismo que la calidad y pertinencia de los contenidos y de los recursos didácticos con los que cuentan, por sólo mencionar algunas variables), se perdió una gran oportunidad de mejorar, efectivamente, la tan mencionada calidad educativa ${ }^{15}$.

Como parte del marco normativo, el gobierno federal ha señalado cuatro atributos que el SEN debe cumplir: el derecho a la asequibilidad, el derecho a la accesibilidad, el derecho en aceptabilidad y el derecho en adaptabilidad. Por los datos hasta ahora explorados, resulta evidente la enorme brecha que separa el discurso oficial del mundo real: ni son claros los programas que se han instrumentado para que las políticas públicas logren garantizar que estos derechos se cumplen, ni tampoco el manejo de los presupuestos que los han acompañado, por más que el discurso oficial haya incorporado el tema de la transparencia y la rendición de cuentas.

Un rápido análisis de los recursos financieros permite afirmar que a pesar de que el ramo presupuestal dedicado a la Educación representa el monto mayor de todos los ramos administrativos del gasto programable del gobierno, el logro educativo no ha mostrado cambios significativos. En efecto, los recursos asignados al rubro educativo aumentan continua e inercialmente y entre 2009 y 2015 han llegado a representar más del $15 \%$ del Presupuesto de Egresos de la Federación (PEF) ${ }^{16}$. Sin embargo, estos montos se han destinado en un gran porcentaje al gasto corriente de la estructura educativa, privilegiando la estabilidad de los líderes sindicales y relegando la inversión en acciones que tengan un mayor potencial para transformar efectivamente la educación.

El propio INEE ha manifestado su preocupación en los siguientes términos:

... el mayor déficit del SEN ocurre sin duda en el terreno de los aprendizajes, ya que una gran proporción de alumnos de la educación obligatoria registra niveles de aprovechamiento insatisfactorio, producto de que los jóvenes no están adquiriendo las competencias necesarias para su adecuado desenvolvimiento en la sociedad, Así lo acreditan los resultados de las pruebas PLANEA como las del Programa para la Evaluación Internacional de Estudiantes (PISA) ${ }^{17}$.

${ }^{15}$ Una espléndida revisión crítica de esta mal llamada «reforma educativa» se encuentra en Ordorika (2016).

16 Véase: Presupuesto de Egresos de la Federación 2009-2015.

${ }^{17}$ INEE, Panorama Educativo de México. Indicadores del Sistema Educativo Nacional 2016 (INEE, 2017: 7). 
En cuanto a infraestructura, las escuelas en peor estado se localizan en zonas marginadas; son precisamente las que atienden a la población vulnerable que, por otro lado, es la más necesitada de una educación de calidad. Son escuelas pobres para pobres. Según la información del Instituto Nacional para la Evaluación de la Educación (INEE), una de cada cinco escuelas tiene techo de lámina de asbesto o de metal, $5 \%$ de las escuelas no tienen paredes o, si las tienen, son de tablaroca, lámina o madera ${ }^{18}$. Esta proporción es 4 veces mayor en escuelas comunitarias y en escuelas indígenas multigrado. Cuatro de cada diez escuelas tienen techos en mal estado, vidrios rotos o estrellados e instalación eléctrica en mal estado; $95 \%$ cuenta con agua, pero no todas tienen baño, a pesar de tener agua; $92 \%$ cuentan con energía eléctrica; una de cada 8 escuelas indígenas multigrado y 2 de cada 5 comunitarias tienen energía eléctrica, pero las otras tres, no.

\section{LAS PERCEPCIONES SOBRE LA EDUCACIÓN}

Con el objeto de conocer algunas características educativas de la población mexicana, lo mismo que sus percepciones sobre la educación, se realizó una encuesta con una muestra representativa a nivel nacional. Para tal fin, se diseñó un cuestionario que, además de una amplia sección sociodemográfica, contó con 55 preguntas dedicadas exclusivamente al tema educativo. Este instrumento fue aplicado a una muestra representativa de 2 mil personas mayores de 15 años, seleccionadas mediante muestreo aleatorio estratificado ${ }^{19}$.

Entre las preguntas que resumen una opinión global de la población entrevistada, está la que pide identificar el principal problema de la educación en México. Casi la mitad de las respuestas coinciden en que no hay escuelas suficientes para todos. Más allá de la seriedad de que la misma población perciba que no hay suficientes escuelas, esto en alguna medida también significa la aceptación implícita de que sus hijos se pueden quedar sin educación. La respuesta que ocupó el segundo lugar en importancia señala al maestro; es decir, se reconoce que los docentes están mal preparados, lo cual es congruente con las respuestas negativas provenientes de otros reactivos en los que se inquiere sobre la asistencia regular de los maestros, sobre si enseñan bien, resuelven dudas y tratan correctamente a los alumnos dentro del aula. Las demás respuestas están distan-

${ }^{18}$ Véase: INEE, La educación obligatoria en México. Informe 2016.

19 Véase: Zubieta, J. et al. (2015) para conocer el cuestionario y los detalles de la encuesta. 
ciadas de las dos primeras, salvo por la que alude al pago de cuotas, a pesar de que la propia Constitución señala que la educación obligatoria es gratuita. Sólo conviene agregar que prácticamente no hay diferencias de género en las percepciones sobre los principales problemas de la educación, salvo en las opciones relacionadas con la operación cotidiana de los planteles, lo cual permite corroborar que en la práctica son las mujeres quienes mantienen una relación más cercana con los procesos escolares de sus descendientes.

\section{CUADRO 3}

¿Cuál cree usted que es el principal problema de la educación en nuestro país?

\begin{tabular}{lccc}
\hline \multirow{2}{*}{\multicolumn{1}{c}{ Principal problema de la educación }} & Total Nacional & \multicolumn{2}{c}{ Sexo } \\
\cline { 3 - 4 } & & Hombre & Mujer \\
\hline No hay escuelas suficientes para todos & 48.4 & 48.4 & 48.4 \\
Los profesores están mal preparados & 46.1 & 46.5 & 45.7 \\
Las cuotas que hay que pagar & 36.9 & 34.7 & 39.3 \\
Los libros de texto y otros materiales para estudiar & 26.4 & 28.0 & 24.9 \\
Los profesores no asisten a clase & 25.6 & 24.2 & 27.1 \\
Las escuelas no tienen buenas instalaciones & 19.5 & 18.6 & 20.5 \\
Falta mucha disciplina y orden en las escuelas & 17.7 & 17.3 & 18.1 \\
No existe suficiente tecnología en las aulas & 15.4 & 16.6 & 14.2 \\
Las instalaciones están en muy mal estado & 12.3 & 13.2 & 11.5 \\
Las prácticas de los sindicatos & 11.8 & 13.2 & 10.3 \\
No hay laboratorios suficientes ni bien equipados & 7.0 & 6.3 & 7.8 \\
No hay suficientes bibliotecas & 3.4 & 3.8 & 3.0 \\
Ninguno & 0.6 & 0.7 & 0.5 \\
NS/NC & 2.1 & 2.7 & 1.9 \\
\hline
\end{tabular}

A pesar de que la calidad de la educación no fue enunciada dentro de los principales problemas de la educación, algunas preguntas del cuestionario estaban formuladas para que los entrevistados expresaran su opinión sobre posibles cambios en ésta en los últimos diez años ${ }^{20}$. Las respuestas indican que muy pocas personas consideran que la calidad ha empeorado; no obstante, vale la pena señalar que son las mujeres quienes menos reconocen que la calidad ha mejorado y, por el contrario, afirman mayormente que

${ }^{20}$ La pregunta estuvo formulada en los siguientes términos: En los últimos 10 años, ¿usted considera que la calidad de la educación ha mejorado, se ha mantenido igual, o ha empeorado? 
se ha mantenido igual o, incluso, que ésta ha empeorado (Gráfico 4). Esto podría deberse a que son las madres quienes mantienen contactos más cercanos con las escuelas a las que van sus hijos que los padres. Como en esta encuesta sólo se entrevistó a población de 15 años y más, entonces es posible suponer que efectivamente las mujeres son más críticas al opinar sobre la calidad educativa, independientemente de si ellas mismas fueron o no excluidas del SEN o de si en el momento de la entrevista tenían hijos en edad escolar.

\section{GRÁFICA 3}

En los últimos 10 años, la calidad de la educación ha...

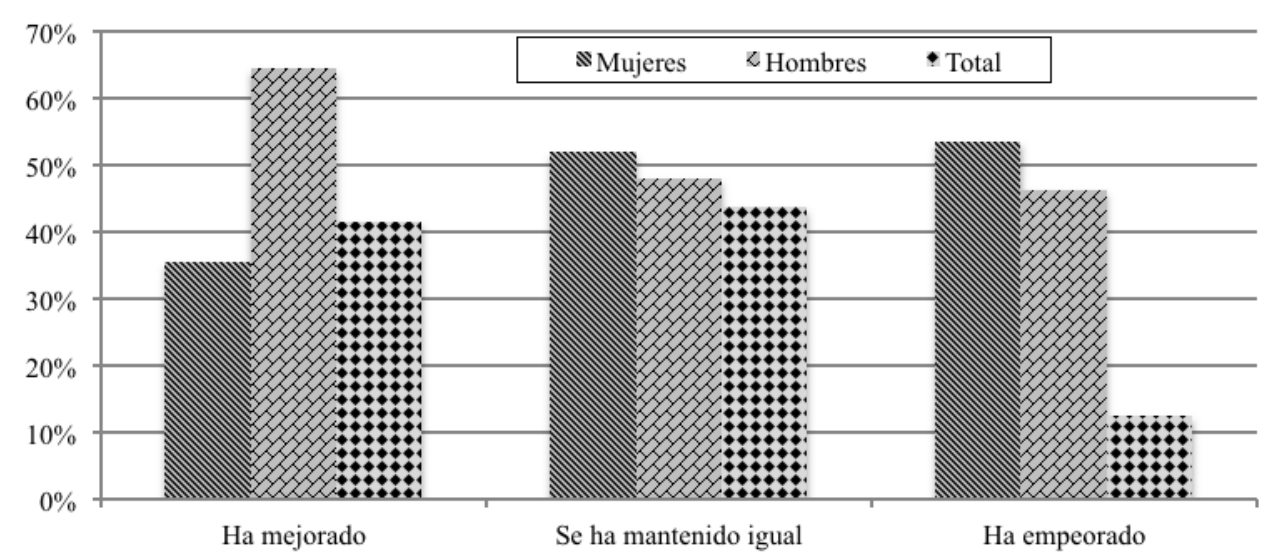

Al reconocer que las opiniones de las personas pueden estar influenciadas por los años transcurridos entre el momento en el que ocurrió un suceso y el presente, el cuestionario incluyó preguntas para poder diferenciar las respuestas en función del tiempo desde que los respondientes dejaron de estudiar. Éste se agrupó en tres segmentos: entre 0 y 5 años, entre seis y diez, y once años o más.

La Gráfica 4 confirma que el tiempo es una variable que interviene y modifica las percepciones de las personas ${ }^{21}$. El Gráfico 5 evidencia que las personas en el grupo 0-5 (es decir, quienes continúan estudiando o dejaron de hacerlo hace cinco o menos años), opinan que la calidad de la educación ha empeorado con mayor frecuencia que quienes

${ }^{21} \mathrm{Si}$ bien se reconoce que las opiniones pueden estar influenciadas por los recuerdos y varían de acuerdo con la memoria de las personas, también debe aceptarse la posibilidad de que haya entre los informantes personas interesadas en temas educativos, ya sea por curiosidad personal, por su ejercicio profesional o por tener hijos en edades escolares. Cualquiera que sea la razón del cambio en las opiniones, en esta encuesta se constató la influencia del tiempo en las percepciones de los encuestados. 


\section{GRÁFICA 4}

En los últimos 10 años, la calidad de la educación ha... (según el tiempo transcurrido desde que dejó la escuela)

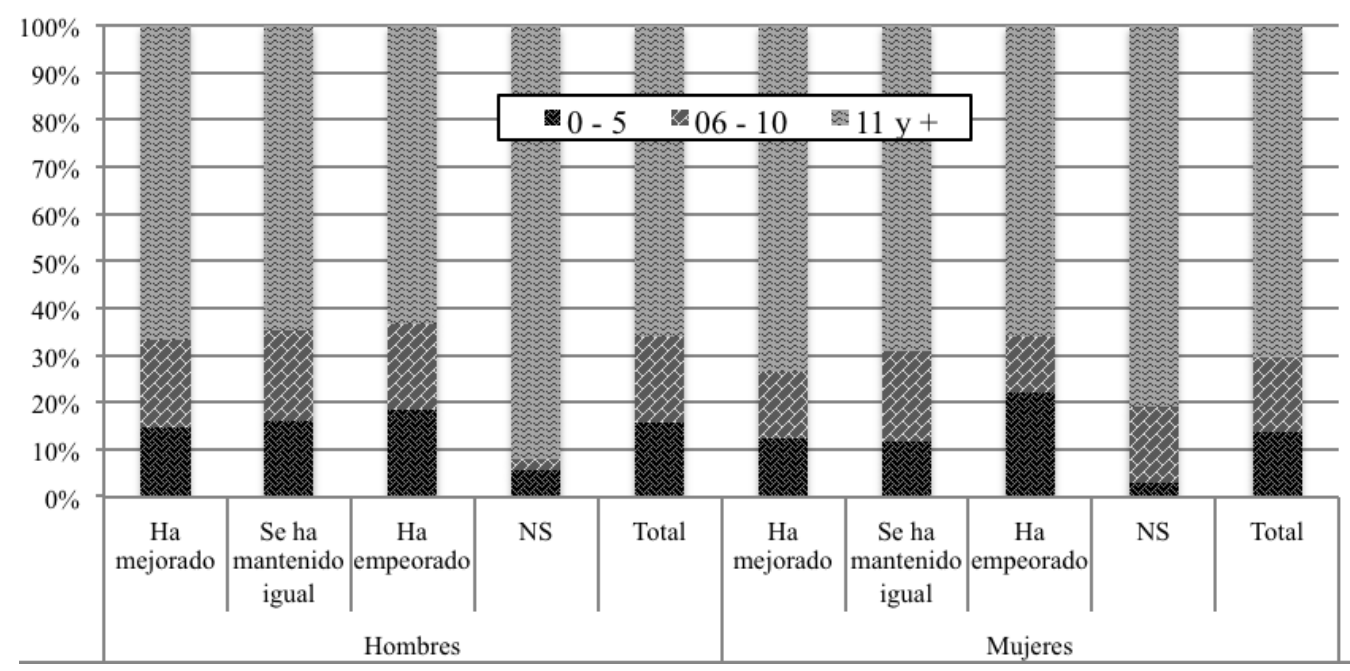

dejaron de estudiar hace más tiempo. Como puede advertirse, la mayoría de la población dejó de estudiar hace once o más años, de ahí que sea el grupo con la no respuesta (NS=No sabe) más numerosa. Pese a ello, nuevamente observamos que la población masculina reporta poca variación en sus opiniones en función del tiempo transcurrido desde que dejó la escuela; por el contrario, pareciera que las opiniones femeninas se endurecen conforme la experiencia educativa se vuelve más reciente.

Lo anterior contrasta con las calificaciones entre 0 y 10 que los entrevistados asignaron a la calidad educativa en México. Los valores relevados permitieron distinguir dos grupos: el de los valores menores a 7 (equivalentes a una calificación reprobatoria) y los que se encuentran entre 7 y 10 (para una calificación aprobatoria). Vale la pena señalar que no se registró ninguna calificación de cero, lo cual pudiera estar relacionado con la escala utilizada que es la que se utiliza para evaluar a los estudiantes.

Si bien la calificación aprobatoria fue mayoritaria para hombres y mujeres, los menores porcentajes se encontraron precisamente entre quienes no han dejado de estudiar o lo hicieron hace menos de seis años. De esta manera, se corrobora que a mayor tiempo transcurrido desde que se deja la escuela, las mujeres son más críticas de la educación y tienden a considerar que la calidad es mala, aunque en el largo plazo, las opiniones de nivelan y la opinión es menos crítica. 


\section{GRÁFICA 5}

Calificación asignada a la calidad educativa, según el tiempo transcurrido desde que dejó la escuela

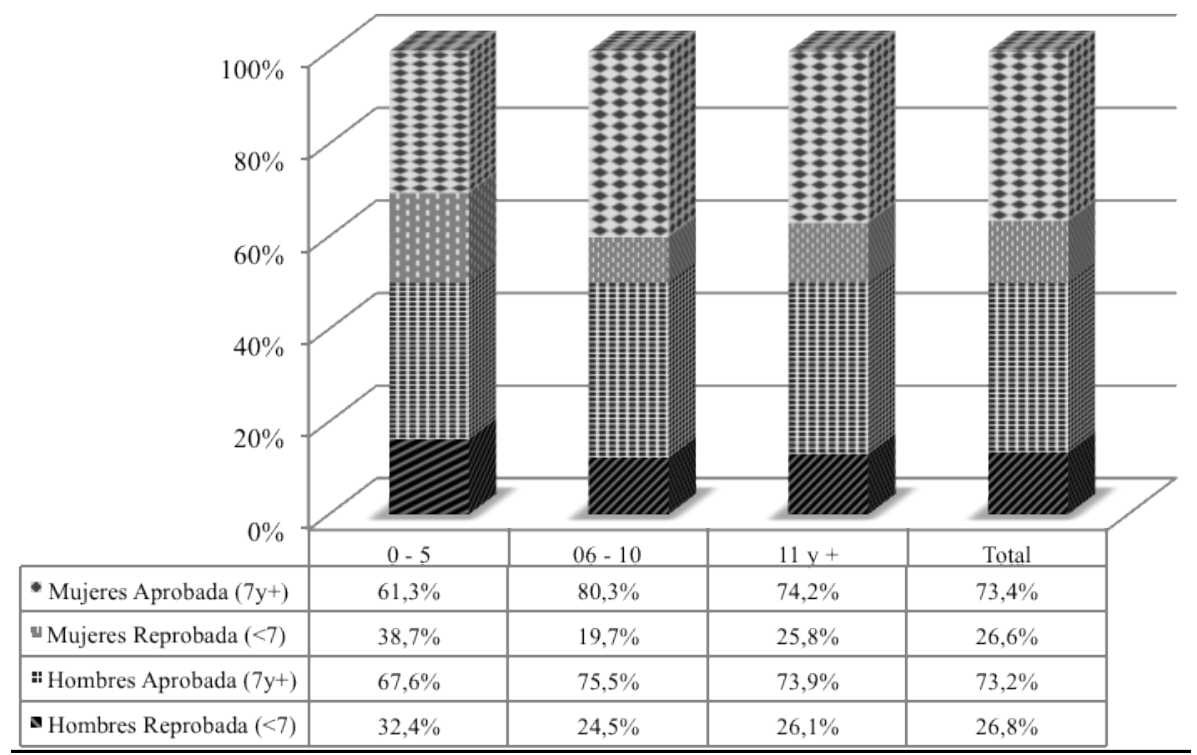

Dado que el segundo problema de la educación referido por la población aludió directamente a los maestros, es conveniente exponer las percepciones manifestadas sobre su desempeño. Al contrastar las opiniones de quienes estudian con las de quienes han dejado de hacerlo, no se perciben grandes diferencias y llama la atención que, a pesar de haberlo reportado como problema, en general la opinión sea buena. Todas las actividades fueron expresadas de manera positiva, de tal suerte que resulta fácil corroborar que los porcentajes relativamente menores están asociados a quienes dejaron de estudiar.

La aparente incongruencia en las opiniones de la población pudiera haber sido generada por los medios de comunicación masiva - especialmente la televisión-que jugaron un papel muy importante en la campaña de desprestigio del magisterio que estaba en su momento cumbre cuando se levantó la encuesta. Este rol infortunado de los medios inicia a partir de la ya comentada «reforma educativa» que, como se dijo anteriormente, atendió más a razones políticas que educativas. 
CUADRO 4

Percepciones sobre el desempeño de los maestros

\begin{tabular}{|c|c|c|c|c|c|c|c|}
\hline \multicolumn{2}{|c|}{$\begin{array}{c}\text { Frecuencia con la que realizan/ } \\
\text { realizaban ... }\end{array}$} & \multirow{2}{*}{$\begin{array}{l}\text { Siempre } \\
31.6\end{array}$} & \multirow{2}{*}{$\begin{array}{l}\begin{array}{c}\text { Frecuen- } \\
\text { temente }\end{array} \\
56.1\end{array}$} & \multirow{2}{*}{$\begin{array}{l}\begin{array}{c}\text { Algunas } \\
\text { veces }\end{array} \\
11.9\end{array}$} & \multirow{2}{*}{$\begin{array}{l}\begin{array}{c}\text { Rara } \\
\text { vez }\end{array} \\
0.5\end{array}$} & \multirow{2}{*}{$\begin{array}{l}\text { Nunca } \\
0.0\end{array}$} & \multirow{2}{*}{$\begin{array}{l}\mathbf{N S} / \\
\mathbf{N C} \\
0.0\end{array}$} \\
\hline Captan/Captaban la & $\begin{array}{l}\text { Quienes } \\
\text { estudian }\end{array}$ & & & & & & \\
\hline $\begin{array}{l}\text { generan/ generaban } \\
\text { interés en la clase }\end{array}$ & $\begin{array}{l}\text { Quienes } \\
\text { han dejado } \\
\text { de estudiar }\end{array}$ & 33.7 & 35.8 & 20.7 & 4.0 & 1.6 & 4.1 \\
\hline \multirow{2}{*}{$\begin{array}{l}\text { Propician/propiciaban } \\
\text { que usted aprenda/ } \\
\text { aprendiera por sí } \\
\text { mismo }\end{array}$} & $\begin{array}{l}\text { Quienes } \\
\text { estudian }\end{array}$ & 38.2 & 38.2 & 19.6 & 1.7 & 2.3 & 0.0 \\
\hline & $\begin{array}{l}\text { Quienes } \\
\text { han dejado } \\
\text { de estudiar }\end{array}$ & 29.2 & 32.5 & 25.7 & 5.5 & 2.6 & 4.4 \\
\hline \multirow{2}{*}{$\begin{array}{l}\text { Son/fueron } \\
\text { respetuosos }\end{array}$} & $\begin{array}{l}\text { Quienes } \\
\text { estudian }\end{array}$ & 52.9 & 35.6 & 11.3 & 0.1 & 0.0 & $0 . .0$ \\
\hline & $\begin{array}{l}\text { Quienes } \\
\text { han dejado } \\
\text { de estudiar }\end{array}$ & 37.5 & 32.8 & 19.0 & 5.6 & 1.9 & 3.2 \\
\hline \multirow{2}{*}{$\begin{array}{l}\text { Generan/generaban } \\
\text { actividades de } \\
\text { aprendizaje para el } \\
\text { desarrollo de su } \\
\text { creatividad }\end{array}$} & $\begin{array}{l}\text { Quienes } \\
\text { estudian }\end{array}$ & 39.8 & 39.4 & 20.1 & 0.2 & 0.6 & 0.0 \\
\hline & $\begin{array}{l}\text { Quienes } \\
\text { han dejado } \\
\text { de estudiar }\end{array}$ & 28.6 & 37.3 & 19.5 & 6.7 & 3.1 & 4.9 \\
\hline \multirow{2}{*}{$\begin{array}{l}\text { Califican/calificaban } \\
\text { las tareas y } \\
\text { actividades escolares } \\
\text { de manera justa }\end{array}$} & $\begin{array}{l}\text { Quienes } \\
\text { estudian }\end{array}$ & 37.7 & 45.5 & 15.6 & 1.1 & 0.0 & 0.0 \\
\hline & $\begin{array}{l}\text { Quienes } \\
\text { han dejado } \\
\text { de estudiar }\end{array}$ & 42.6 & 29.8 & 17.1 & 5.2 & 1.2 & 4.0 \\
\hline \multirow{2}{*}{$\begin{array}{l}\text { Asisten/asistían } \\
\text { puntualmente a la } \\
\text { escuela }\end{array}$} & $\begin{array}{l}\text { Quienes } \\
\text { estudian }\end{array}$ & 42.3 & 36.8 & 20.3 & 0.5 & 0.0 & 0.1 \\
\hline & $\begin{array}{l}\text { Quienes } \\
\text { han dejado } \\
\text { de estudiar }\end{array}$ & 39.4 & 32.2 & 17.5 & 4.9 & 1.2 & 4.7 \\
\hline
\end{tabular}


Aún cuando sus habitantes reconocen que hay problemas en el SEN — y a pesar de que no se respeta su derecho a la educación-, el país puede seguir apostando por un mejor futuro si logra que la educación se convierta en el motor del cambio anhelado. Lo anterior se fundamenta en que la población, independientemente de su edad, reconoce dentro de las motivaciones por estudiar el deseo de superarse, y en las instituciones educativas, los espacios donde es posible aprender. Aunque ya no asista a la escuela, e independientemente de las razones por las que dejó de hacerlo, las y los mexicanos siguen teniendo un alta estima por la educación, al tiempo que continuán relacionándola con procesos de aprendizaje y con mejores posibilidades para ubicarse en el mercado de trabajo.

\section{CUADRO 5}

El valor de estudiar: motivaciones

\section{Motivaciones para estudiar}

\begin{tabular}{lccc}
\hline \multirow{2}{*}{ Motivaciones para estudiar } & \multicolumn{3}{c}{ Edad } \\
\cline { 2 - 4 } & $\mathbf{1 5 - 2 4}$ años & $\mathbf{2 5}$ a $\mathbf{3 4}$ años & $\mathbf{3 5}$ a $\mathbf{4 4}$ años \\
\hline Porque deseo superarme & 70.5 & 72.2 & 70.5 \\
Porque quiero tener un mejor trabajo o un mejor & 9.1 & 8.9 & 6.9 \\
sueldo & & & \\
Porque deseo aprender & 7.9 & 15.6 & 9.7 \\
Porque me gusta estudiar & 6.2 & 0.0 & 0.0 \\
Porque para hacer lo que yo quiero hacer, & 4.7 & 0.0 & 13.0 \\
necesito estudiar & & & \\
Porque tengo una escuela cerca & 1.3 & 0.0 & 0.0 \\
Porque mis papás me lo exigen & 0.3 & 0.0 & 0.0 \\
Porque me puede dar prestigio & 0.0 & 3.3 & 0.0 \\
\hline
\end{tabular}

Con el afán de profundizar en la relación que la población construye entre escuela y mercado laboral, se incluyeron varios reactivos. A partir de las respuestas se puede afirmar que, efectivamente, esta relación no ha sufrido cambios en el imaginario colectivo. La mayoría de la población reconoce los beneficios de estudiar, aunque no lo haga.

No se ignora la posibilidad de que al interior de las familias se hayan diseñado estrategias de sobrevivencia que privilegian - especialmente en el caso de los varonesla posibilidad de encontrar o tener un empleo por sobre la inversión de tiempo que implícitamente conlleva la permanencia en la escuela, con la consecuente ausencia de ingresos. 


\section{CUADRO 6}

Creencias sobre la educación

\begin{tabular}{lcccc}
\hline \multicolumn{1}{c}{ ¿Cree usted que...? } & Sí & No & NS & NC \\
\hline La escuela prepara para tener mejores empleos & 84.4 & 12.6 & 2.3 & 0.7 \\
La escuela es una opción para todos & 82.4 & 16.0 & 1.0 & 0.6 \\
Estudiar mejora el ingreso de las personas & 82.1 & 14.3 & 2.8 & 0.8 \\
Por estudiar tenemos una mejor sociedad & 77.5 & 17.2 & 4.4 & 0.9 \\
En la escuela hay cupo para todos & 46.6 & 48.9 & 3.9 & 0.6 \\
\hline
\end{tabular}

El Cuadro 6, sin embargo, revela que la escuela es considerada una opción para todos; es decir que, a pesar de que muchos ignoran que tienen como un derecho a la educación, la población sabe que no todos han tenido oportunidad de escolarizarse y que no hay escuelas suficientes, seguramente porque todos deberían tener esa opción.

Para matizar esta interpretación optimista, asociada con la opinión casi generalizada de que estudiar mejora el ingreso de las personas, a continuación se muestran las respuestas a la pregunta sobre las razones por las que se interrumpieron los estudios, formulada a quienes así lo declararon. Más allá de que las personas de 35 años y más consideran mayoritariamente que la edad es una razón para dejar de estudiar, llama también la atención que la disponibilidad de tiempo sea otra de las razones más frecuentemente declaradas entre población con menor edad, junto con algunas características específicas del grupo más joven que, en plena edad escolar, reconoce que el proceso educativo tiene un alto grado de dificultad y, además, es aburrido.

Más allá de los sesgos que estas opiniones pueden tener, de manera implícita ponen en duda la capacidad de las y los maestros para hacer que los procesos de enseñanza y de aprendizaje sean generadores de talento y de creatividad. Desde luego, no se puede asumir que esto sea responsabilidad exclusiva del docente; poco puede hacer el magisterio si las condiciones y los espacios en los que enseñan no cumplen con los requisitos mínimos indispensables para hacer del aprendizaje un proceso activo de descubrimiento y aprendizaje. 
CUADRO 7

Razones para no seguir estudiando según grupos de edad

\begin{tabular}{lrrrrrr}
\hline \multicolumn{1}{c}{ Razones } & \multicolumn{7}{c}{ Edad } \\
\cline { 2 - 7 } & $\mathbf{1 5 - 2 4}$ & $\mathbf{2 5 - 3 4}$ & $\mathbf{3 5 - 4 4}$ & $\mathbf{4 5 - 5 4}$ & $\mathbf{5 5 - 6 4}$ & $\mathbf{6 5}$ y más \\
\hline Porque es muy aburrido & 24.7 & 1.6 & 1.2 & 2.5 & 0.0 & 0.0 \\
Porque ya estoy satisfecha/o con lo & 24.2 & 27.0 & 18.8 & 12.3 & 9.5 & 5.7 \\
que tengo & & & & & & \\
Porque es muy difícil & 19.3 & 10.0 & 15.4 & 7.2 & 3.0 & 3.4 \\
Porque no tengo tiempo & 17.7 & 28.2 & 24.4 & 9.2 & 6.2 & 0.0 \\
Por mi edad & 6.3 & 22.1 & 33.3 & 63.3 & 80.9 & 76.5 \\
Porque odio los exámenes & 3.3 & 3.3 & 2.1 & 2.4 & 0.0 & 10.4 \\
Porque no sirve lo que enseñan & 3.3 & 3.3 & 0.0 & 0.6 & 0.0 & 0.0 \\
Porque no me queda cerca ninguna & 1.2 & 2.1 & 1.7 & 1.0 & 0.4 & 0.0 \\
escuela & & & & & & \\
NS/NC & 0.0 & 2.3 & 3.1 & 1.4 & 0.0 & 3.9 \\
\hline
\end{tabular}

Otra posible explicación de estas respuestas se encuentra en los contenidos curriculares y en la organización misma del SEN, temas sobre los cuales hace décadas que no se propicia una discusión, como la que ameritan. Dada la dificultad inherente a preguntar por la pertinencia del curriculum, los reactivos se enfocaron a indagar si las y los entrevistados encontraban o no alguna relación entre lo que aprendieron en la escuela y el trabajo, desagregando las respuestas en función de su nivel escolar.

La Gráfica 6 muestra que, a mayor nivel escolar, mayor es la percepción de que efectivamente hay una relación directa entre lo que se aprende en la escuela y el trabajo que se desempeña. A menor escolaridad, mayores son las dudas sobre esta relación, aunque hombres y mujeres con educación básica (preescolar, primaria y secundaria) pongan en tela de juicio si existe esta relación o, en caso afirmativo, se inclinan por señalar que ésta es débil. Es un hecho que las percepciones cambian conforme se avanza en el SEN; aunque nadie encuentra una relación estrecha entre las dos esferas, la población con educación media superior acepta que existe esa relación.

Aunque las diferencias de género sean pequeñas, resulta interesante advertirlas porque evidencian que los varones son más críticos que las mujeres del tipo de relación que pudiera existir entre la escuela y el empleo.

Como se ha venido sugiriendo en este artículo, es inexplicable que no hayan habido cambios ni cuestionamientos sobre los contenidos y la estructura del SEN que pudieran responder, ya no a las necesidades del mundo laboral, sino a los conocimientos, habili- 
GRÁFICA 6

Relación entre lo que aprendió en la escuela y su trabajo

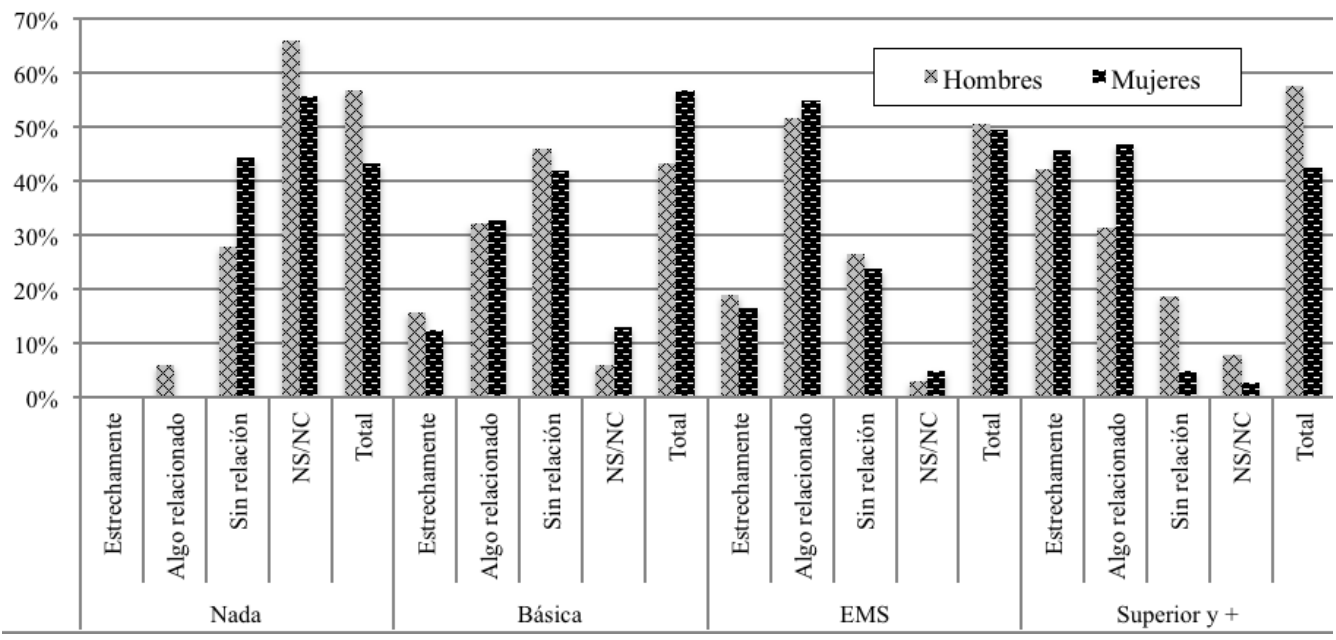

dades y valores que debiera tener un curriculum del siglo XXI en un país tan diverso como inequitativo como México. Temas como el cuidado del ambiente, valores como la tolerancia a la diversidad y otros contenidos que hoy en día son imprescindibles, en el futuro se tornarán ineludibles y, pese a ello, todavía no se contempla su inclusión.

Precisamente por esta aparente falta de conexión entre la educación y un futuro laboral ya imaginable, en la encuesta también se solicitó la opinión sobre la relación entre lo aprendido y el trabajo que se desempeñará en el futuro, sin definir plazos para que éste ocurra.

Nuevamente, los resultados que se muestran en la Gráfica 7 permiten corroborar la visión el deseo de la población en el sentido de imaginar un futuro en el que habrá una mayor relación entre lo que se aprende en la escuela y el trabajo. Similar a lo expuesto en la gráfica anterior, a mayor escolaridad, mayor el número de personas que encuentran que la relación será estrecha. Por su parte, las mujeres con menos escolaridad (educación básica y media superior) se muestran un poco más cautas al opinar con mayor frecuencia que ambas esferas no estarán estrechamente sino algo relacionadas.

Al relacionar estos resultados con las opiniones expresadas sobre la calidad de la educación, pareciera plausible suponer que si las mujeres son quienes perciben que la calidad de la educación no ha mejorado en los últimos años, tampoco lo hará notoriamente en los siguientes. Esta hipótesis deberá ser corroborada; sin embargo, permite suponer que ésta sea una de las razones por las que no alcanzan a ver con optimismo la relación estudio-trabajo. 


\section{GRÁFICA 7}

Relación entre lo que aprendió en la escuela y su trabajo futuro

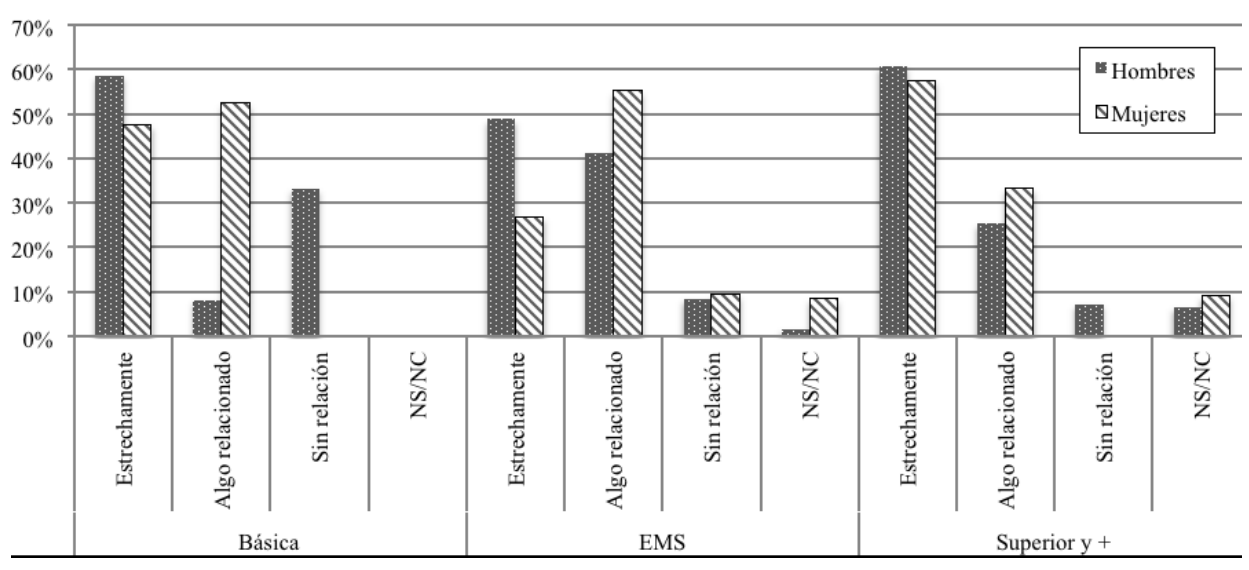

Otro factor que pudiera estar operando en las percepciones de la población es la dificultad para conseguir empleo. Numerosos estudios han demostrado que tanto el empleo como la calidad del empleo han disminuido. Las políticas gubernamentales en la materia han sido pobres, como también lo han sido las que atienden al desarrollo social y económico. La población mexicana no ha podido romper los círculos intergeneracionales de pobreza, producto de la baja movilidad social que registra el país ${ }^{22}$.

Al indagar sobre las dificultades para conseguir empleo, no debe extrañar que la población se divida prácticamente en dos grupos: quienes consideran que no es difícil y quienes piensan que sí lo es, aunque esa afirmación esté matizada (sí en parte).

\section{GRÁFICA 8}

Percepción de las dificultades para conseguir empleo

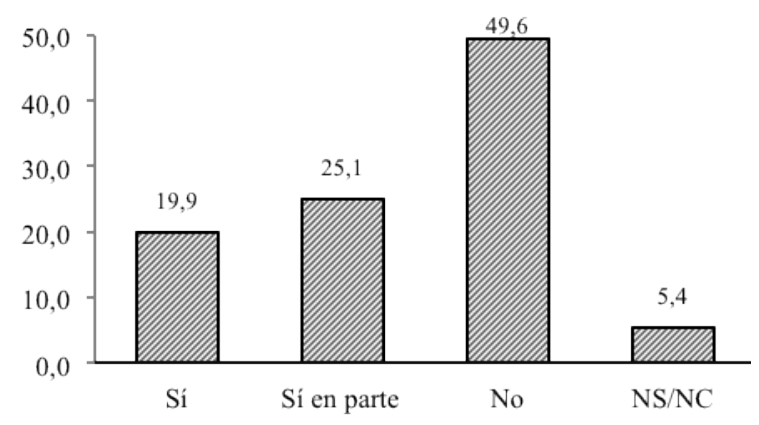

${ }^{22}$ Véase: Encuesta de Movilidad Social (EMOVI) en México 2017 (CEEY, 2017). 
Dadas las características de escolaridad que presenta la población y considerando las dificultades económicas por las que ha venido atravesando el país, no debe de llamar la atención que la mitad de la población enfrente problemas para conseguir trabajo.

$\mathrm{Si}$ a lo anterior se agrega la falta de relación que las personas ven entre lo que se aprende en la escuela con lo que demanda el mercado laboral, pocas son las oportunidades que tiene el país para lograr que la población vuelva a las aulas o se acerque a ellas por primera vez. Por más valor que las y los mexicanos asignen a la educación, por más que le reconozcan su potencial para mejorar las condiciones que promuevan un mayor desarrollo, difícil resulta pensar que estas condiciones y actitudes cambiarán en el futuro cercano.

\section{CUADRO 8}

Aspectos para mejorar la educación en México

\begin{tabular}{lccc}
\hline \multicolumn{1}{c}{ Aspectos a atender } & $\begin{array}{c}\text { Total } \\
\text { Nacional }\end{array}$ & \multicolumn{2}{c}{ Sexo } \\
\cline { 2 - 4 } & Total & Hombres & Mujeres \\
\hline Capacitar mejor a los profesores & 60.2 & 59.8 & 60.5 \\
Construir más escuelas & 56.9 & 56.1 & 57.6 \\
Mejorar las instalaciones de todas las escuelas & 36.4 & 37.9 & 34.9 \\
Destinar más recursos a la educación & 33.8 & 33.0 & 34.5 \\
Comprar más computadoras y otras tecnologías & 24.5 & 25.1 & 23.9 \\
Mejorar las bibliotecas para que tengan muchos más libros & 17.6 & 17.7 & 17.5 \\
Resolver los problemas de los sindicatos & 16.7 & 16.6 & 16.8 \\
Capacitar bien a los directores & 14.7 & 15.7 & 13.9 \\
Disminuir el porcentaje de alumnos que dejan la escuela & 13.9 & 13.6 & 14.2 \\
Otro & 0.8 & 0.7 & 0.9 \\
NS/NC & 2.8 & 2.9 & 2.6 \\
\hline
\end{tabular}

Finalmente, el Cuadro 8 presenta las respuestas recibidas cuando se pidió a los entrevistados que enunciaran tres aspectos que el gobierno debe atender para mejorar la educación en el país. Congruente con la problemática identificada en un principio, la población señala la capacitación de los docentes y la construcción de escuelas para mejorar la calidad y dar cabida a todos quienes, por falta de espacios o de equipamiento, se quedan fuera. A pesar de que es posible que la población no conozca la magnitud de los recursos que año con año se destinan a la educación, se manifiesta por aumentar el pre- 
supuesto porque no es difícil suponer que con más recursos seguramente se tendrán mejores condiciones para atender a la demanda. En franco reconocimiento del papel que la tecnología tiene en la vida cotidiana, el quinto lugar lo tiene la compra de equipos de cómputo y otras tecnologías, para lo cual también se requieren mayores recursos.

\section{DISCUSIÓN}

Una encuesta nacional, realizada en hogares a personas de 15 años y más, nos ha permitido constatar las grandes paradojas de este sistema que ha sido fundamentalmente excluyente y discriminador. Pese a ello, los mexicanos percibimos que la falta de logros educativos está más directamente relacionada con decisiones y situaciones individuales que con las grandes disparidades que existen en el acceso a la educación.

Desde sus orígenes, México ha estado marcado por una inequitativa distribución del ingreso que se ha traducido en oportunidades educativas muy desiguales para grandes segmentos de su población. El análisis de la exclusión educativa involucra la revisión de algunos estudios que abordan temas como el de la pobreza y la desigualdad, a la par de requerir de la profundización en el conocimiento de las características en las que opera un sistema educativo; en función de todo ello es posible deducir algunas consecuencias en el terreno del empleo y de la precariedad laboral (Donoso y Schiefelbein, 2007).

Si bien el SEN no ha asumido entre sus objetivos explícitos preparar a los futuros técnicos, empleados, líderes o emprendedores de los sectores público y privado, de acuerdo al INEE sí contempla el desarrollo «de los valores democráticos, los valores naconales y se fomenta el mejoramiento de la convivencia humana, a través del desarrollo de los ideales de fraternidad e igualdad de los hombres, evitando los privilegios de razas, de religión, de grupos, sexos o de individuos» ${ }^{23}$.

De lo anterior se desprende que si la educación ha de servir para construir una sociedad más democrática, más próspera y menos desigual, queda mucho camino por andar y muchas contradicciones que superar para que el SEN tenga todos los instrumentos necesarios y los aplique, de tal suerte que esté en condiciones de garantizar mayor equidad en el acceso a una educación de calidad.

A pesar de que el discurso oficial de las autoridades educativas mexicanas adoptó la Declaración Mundial sobre Educación para Todos, aprobada en 1990 por la Conferencia

${ }^{23}$ Véase: INEE (2005; 17), Políticas y sistemas de evaluación educativa en México. Avances, logros y desafios. 
Mundial sobre educación para Todos (Jomtien, Tailandia, marzo de 1990), persisten mecanismos que han impedido de facto este propósito.

Por todo lo anterior, resulta paradójico que la población mexicana no se perciba como excluida de un sistema que, por un lado, establece la obligatoriedad de la educación pero, por el otro, no le garantiza las condiciones necesarias para su permanencia. En general, como se demostró, los mexicanos consideran que la educación favorece la movilidad social, aunque las evidencias recientes indiquen que este efecto se ha ido reduciendo y, en la realidad, así lo empiecen a experimentar algunos sectores de la población.

Las opiniones de que es más fácil que un varón encuentre trabajo con menor escolaridad que una mujer pronto tendrán que ser revisadas, dado que la población femenina, a pesar de la condición de marginación que todavía prevalece en ciertos grupos sociales, exhibe un mejor desempeño escolar. Pese a ello, el abandono escolar femenino es elevado, especialmente en los periodos de transición entre los diferentes niveles. Si bien es necesario realizar investigaciones rigurosas para demostrarlo, pareciera que este fenómeno está más directamente asociado a las edades en las que la mujer inicia su vida fértil que a un rechazo individualizado, independientemente de la causa que lo pudiera generar (como el bullying, el acoso, la oposición de los padres o de la pareja, las condiciones y valores de la familia, etc.). Estas situaciones permiten suponer que los problemas de embarazo adolescente — presentes en la sociedad desde hace algunos añospodrían estar interviniendo, junto con las provenientes de las necesidades de contar con un ingreso, en la decisión de las jóvenes de interrumpir los estudios, independientemente de si piensan retomarlos más adelante.

¿Cómo rediseñar un Sistema Educativo que asegure a la población excluida que origen no es destino y que la convenza de que mediante la educación se verán mejor capacitados para pensar en estrategias de sobrevivencia? ¿Cómo garantizar que una política educativa correcta puede ir de la mano con otra de empleo digno que permita aspirar a mejores salarios para una población que todavía mantiene la esperanza en la educación como motor de cambio?

Douglas B. Downey y Dennis J. Condron (2016: 234) plantean una pregunta que se antoja pertinente en el presente análisis: ¿se lograría avanzar más si las brechas de desempeño escolar debidas a las condiciones socioeconómicas y raciales se redujeran mediante la reforma de las escuelas, o mediante reformas de los contextos sociales y económicos en los que las escuelas se ubican?

Al modificar el contexto en el que vive y se desarrolla la población, la educación también ha de modificar actitudes, valores y tradiciones. Pese a la buena imagen que, en términos generales, tiene la educación, la tercera parte de los mexicanos no lee libros y 
al menos uno de cada cuatro no consulta los periódicos. Investigaciones recientes han demostrado que los ambientes educativos menos favorecidos son los que no fomentan ni cultivan el valor del estudio. De manera opuesta, el origen social influye sobre los tipos de inclusión educativa de ciertos grupos sociales y son precisamente esos tipos de inclusión los que pueden llegar a determinar la permanencia de los individuos en el sistema educativo. De ahí que se vuelva impostergable el reconocimiento de la existencia de diversas formas de inclusión y de exclusión educativa, aunque no formen parte del imaginario colectivo; sin lugar a dudas, esto tendrá impactos favorables para la población.

\section{BIBLIOGRAFÍA}

Blanco, B. E. (2012). «El reciclaje de la desigualdad: exclusiones educativas en América Latina», en Puchet, M. et al., América Latina en los albores del siglo XX: 2. Aspectos sociales y políticos. México, Flacso México.

Centro de Estudios Espinosa Yglesias (CEEY; 2017). Encuesta de Movilidad Social en México 2017. Disponible en: http://www.ceey.org.mx/encuesta/levantamiento-encuesta-esru-movilidad-social-mexico-2017-emovi-2017

Donoso, S. y E. Schiefelbein (2007). «Análisis de los modelos explicativos de retención de estudiantes en la Universidad: una visión desde la desigualdad social», Estud. pedagóg. [online], Vol. 33, n. 1, pp. 7-27. Disponible en: http://www.scielo.cl/scielo. php?script=sci_arttext\&pid=S0718-07052007000100001

Downey, D. y D. Condron (2016). «Two Questions for Sociologists of Education: A Rejoinder», Sociology of Education, 89(3), 234-235.

Fraustro, A. (2017). «El rezago educativo total y su atención en México». Revista Latinoamericana de Estudios Educativos (México), Vol. XLVII (2). Disponible en: http://www.redalyc.org/html/270/27052400003/

Holland, J. (2009). «Young people and social capital. Uses and abuses?» Young Nordic Journal of Youth Research, SAGE Publications. Vol. 17(4): 331-350.

Inee (2005), Políticas y sistemas de evaluación educativa en México. Avances, logros y desafios. México. Disponible en:

http://publicaciones.inee.edu.mx/detallePub.action?clave=P1D206

Inee (2014). El derecho a una educación de calidad. Informe 2014. Disponible en:

http://publicaciones.inee.edu.mx/buscadorPub/P1/D/239/P1D239.pdf

Inee (2015). Panorama Educativo de México. México.

Inee (2016a). La educación obligatoria en México. Informe 2016. México. 
Inee (2016b). Panorama Educativo de México. México.

Inee (2017). Directrices para mejorar la atención educativa de niñas, niños y adolescentes indígenas. México. Disponible en:

http://publicaciones.inee.edu.mx/buscadorPub/P1/F/104/P1F104.pdf

Ordorika, I. (2016). Transcripción de la ponencia presentada en el Foro Hacia una verdadera Reforma Educativa, Por México Hoy, México, pp. 31-40. Disponible en: https://pormxhoy.org/descargas/1487191324-TranscForoEdu.pdf

Organización para la Cooperación y el Desarrollo Económico, OCDE (varios años). Education at a glance. OECD Indicators. D.E.: http://www.oecd.org/edu/education-at-a-glance-19991487.htm

Organización de Estados Iberoamericanos (OEI). Plan Iberoamericano para la Alfabetización y Educación Básica de Personas Jóvenes y Adultas 2007-2015. Madrid, 2012. DE: http://oei.es

Rodríguez-Gómez, R. e I. Ordorika (2012). «Cobertura y estructura del Sistema Educativo Mexicano: problemática y propuestas» [en] Narro Robles, J., J. Martuscelli y E. Bárzana (Coords.), Plan de diez años para desarrollar el Sistema Educativo Nacional. UNAM, México. Disponible en: http://www.planeducativonacional.unam.mx/ PDF/CAP_07.pdf

SEP (2016). Estadística del Sistema Educativo. México. Ciclo Escolar 2015-2016. Disponible en: http://www.snie.sep.gob.mx/descargas/estadistica_e_indicadores/ estadistica_e_indicadores_educativos_15MEX.pdf

UNESCO (2011). Creating and Sustaining Literacy Environments, UNESCO. DE: http://unesdoc.unesco.org/images/0021/002146/214653e.pdf

UNESCO (2016). Informe de Seguimiento de la Educación en el Mundo. La Educación para Todos 2000-2015: Logros y Desafios. D.E.: http://es.unesco.org/gem-report/ report/2015/la-educaci\%C3\%B3n-para-todos-2000-2015-logros-y-desaf\%C3\%ADos\#sthash.uacfmBC6.dpbs

Zubieta, J., F. Gamboa et al. (2012). «Mejora de la calidad educativa» [en] Narro Robles, J., J. Martuscelli y E. Bárzana (Coords.), Plan de diez años para desarrollar el Sistema Educativo Nacional. UNAM, México, 2012, pp. 349-370. ISBN 978-60702-3911-3. Disponible en: http://www.planeducativonacional.unam.mx/PDF/ CAP_14.pdf

Zubieta, J, T. Bautista, et al., (2015). Educación. Las paradojas de un sistema excluyente. Col. «Los Mexicanos vistos por sí mismos. Los grandes temas nacionales». Instituto de Investigaciones Jurídicas, UNAM. México, 2015, 351 pp. ISBN 978-60702-6987-5. Disponible en: http://www.losmexicanos.unam.mx./educacion/index. html 